\title{
Role of continuous hypertonic saline in acute ischemic infarcts: a systematic literature review
}

\section{REVIEW ARTICLE}

Arunit J. S. Chugh, MD ${ }^{1,2}$; Marquis Maynard, BS²; Kerrin Sunshine, BS ${ }^{2}$; Berje H. Shammassian, MD, MPH ${ }^{1,2}$; Adam Sauer, MD²; Kolade Odetoyinbo, $\mathrm{BS}^{2}$; S. Alan Hoffer, $\mathrm{MD}^{1,2}$

'Department of Neurological Surgery, University Hospitals Cleveland Medical Center, Cleveland, $\mathrm{OH}, \mathrm{USA}$

${ }^{2}$ Case Western Reserve University School of Medicine, Cleveland, OH, USA
Neurocritical

\section{Care}

Received: February 6, 2021

Revised: February 26, 2021

Accepted: March 13, 2021

Corresponding Author:

Arunit J. S. Chugh

Department of Neurological Surgery,

University Hospitals Cleveland Medical

Center and Case Western Reserve

University School of Medicine, 11100

Euclid Avenue HAN 5042, Cleveland,

$\mathrm{OH}$ 44106, USA

Tel: +1-216-844-3472

Fax: +1-216-844-3014

E-mail: arunit.chugh@uhhospitals.org

Continuous hypertonic saline for hemispheric ischemic infarcts has been routinely used in neurocritical centers for the management of malignant cerebral edema. However, the data supporting its use are extremely limited. We present a systematic literature review that highlights five studies (one randomized control trial and four retrospective cohort) where the effects of continuous hypertonic saline were studied in patients with acute ischemic infarcts. Collectively, there is a lack of substantial evidence supporting its use. Also, this review emphasizes significant study flaws that make the conclusions largely nongeneralizable. Although the reported studies demonstrate improvement in control of intracranial pressure, there are no significant differences in neurological or functional outcomes or overall mortality.

Keywords: Brain edema; Saline solution, hypertonic; Ischemic strokes; Mortality; Critical care outcomes

\section{INTRODUCTION}

Malignant cerebral edema is a general term that describes pathologic swelling of the brain parenchyma and can be the result of influx of fluid into the extravascular space. In the setting of acute ischemic infarcts, cytotoxic edema results from cellular death and resultant intracellular influx of water. Although this is thought to be the mechanism of early cerebral edema in ischemic infarcts, later stages likely involve a loss of the integrity of the blood-brain barrier as well $[1,2]$. Cerebral edema is directly correlated with increased intracranial pressure (ICP) and has been determined to be an independent marker of poor outcomes in patients with stroke [3]. Malignant middle cerebral artery (MCA) syndrome refers to severe cerebral edema and subsequent neurologic decline that occurs as a result of a large MCA territory infarct.

There are several mechanisms by which hypertonic therapy has been postulated to decrease cerebral edema and subsequently ICP. Increasing osmolality in the intravascular space produces an osmotic gradient and draws fluid out of the extravascular spaces; by decreasing the viscosity in the intravascular space, there is a reflexive vasoconstriction that reduces cerebral blood flow. However, these mechanisms may only decrease ICP transiently [2]. Also,

(C) 2021 The Korean Neurocritical Care Society

This is an Open Access article distributed under the terms of the Creative Commons Attribution Non-Commercial License (http://creativecommons.org/licenses/by-nc/4.0/) which permits unrestricted noncommercial use, distribution, and reproduction in any medium, provided the original work is properly cited. 
the eventual loss of autoregulation that occurs in ischemic infarcts means loss of the blood brain barrier and resultant efflux of the hyperosmolar contents, which could theoretically increase cerebral edema [1].

The role of continuous hypertonic saline infusion for control of malignant cerebral edema is controversial. Despite its widespread use in clinical practice, the data supporting its use over intermittent boluses dosing are limited. Although continuous infusion of hypertonic saline has been effective in reducing cerebral edema in animals, limited data was found in humans [4-7]. In this systematic literature review, we present the current data on the use of continuous hypertonic saline. Our objectives were to determine the mortality and neurological outcomes when continuous hypertonic saline is used in the management of malignant cerebral edema secondary to acute ischemic infarcts and to determine the rates of systemic complications, hospital length of stay, and functional outcomes in patients in whom continuous hypertonic saline was used.

\section{METHODOLOGY}

A systematic literature review of the use of continuous hypertonic saline in the setting of malignant cerebral edema secondary to acute ischemic infarcts was performed in accordance with guidelines for the preferred reporting items for systematic review and meta-analysis (PRISMA) [8]. A search strategy using controlled vocabulary, in combination with keywords, pertaining to administration of hypertonic saline in acute ischemic infarcts was performed by a health science librarian via the following electronic databases: Medline via PubMed, Ovid, Embase via Embase.com, the Cochrane Library (Wiley interface, current issue), Web of Science/Knowledge via Clarivate Analytics, ClinicalTrials.gov, and Scopus. The search was limited to English language literature. The references of the searched literature were utilized to expand the search and include all grey literature.

Data Management was conducted through Rayyan online services [9]. Abstracts obtained under previously described platforms were reviewed independently by the following authors for relevance: AJSC, MM, and KS. The eligible studies were then independently reviewed in full to determine adherence to inclusion and exclusion criteria by these same authors. Nonduplicated studies were then reviewed by AJSC for appropriateness. AJSC and MM then utilized the Newcastle-Ottawa Assessment of Quality for evaluation of the quality of the included studies.

Inclusion criteria were the following: randomized controlled trials, prospective and retrospective cohort studies, and database studies in English language; Adult patients $\geq 18$ years old with acute ischemic infarcts (including anterior and posterior circulation) in whom hypertonic saline was administered. Exclusion criteria were the following: reviews, meta-analysis, animal studies, studies involving pediatric patients ( $<18$ years of age), traumatic brain injury studies, and non-English language studies.

The initial intended primary outcome was mortality rate (overall and/or neurological death defined by death secondary to brain herniation, refractory elevated ICP, or direct involvement of vital neurologic structures) among patients that received hypertonic saline as means of reducing cerebral edema. Secondary outcomes were the following: (1) neurological outcomes (Glasgow Outcome Scale) among patients that received continuous hypertonic saline, (2) rates of adverse events (i.e., congestive heart failure, pulmonary edema, and acute kidney injury), (3) hospital and intensive care unit length of stay, and (4) functional outcomes (modified Rankin Scale).

\section{RESULTS}

Eight hundred ninety-nine records were initially identified. After initial screening, 154 abstracts were reviewed. After further application of exclusion criteria, 14 full-length articles were reviewed. However, of these studies, only five involved continuous administration of hypertonic saline and were thus included for data synthesis (Fig. 1, Table 1) [10-14]. The overall quality of the studies as determined by the Newcastle-Ottawa scale ranged from fair to good, and most were limited by follow-up length and comparability of the study groups (Table 2) [10-14].

In 1998, Qureshi et al. [10] conducted a single-center retrospective review of 27 patients with cerebral edema, six of whom

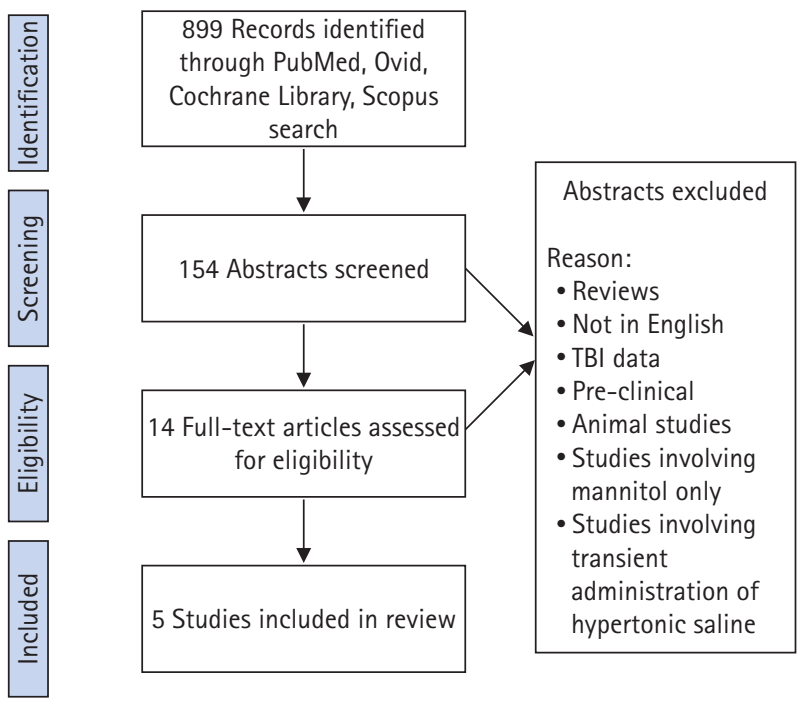

Fig. 1. Study flowchart. TBI, traumatic brain injury. 


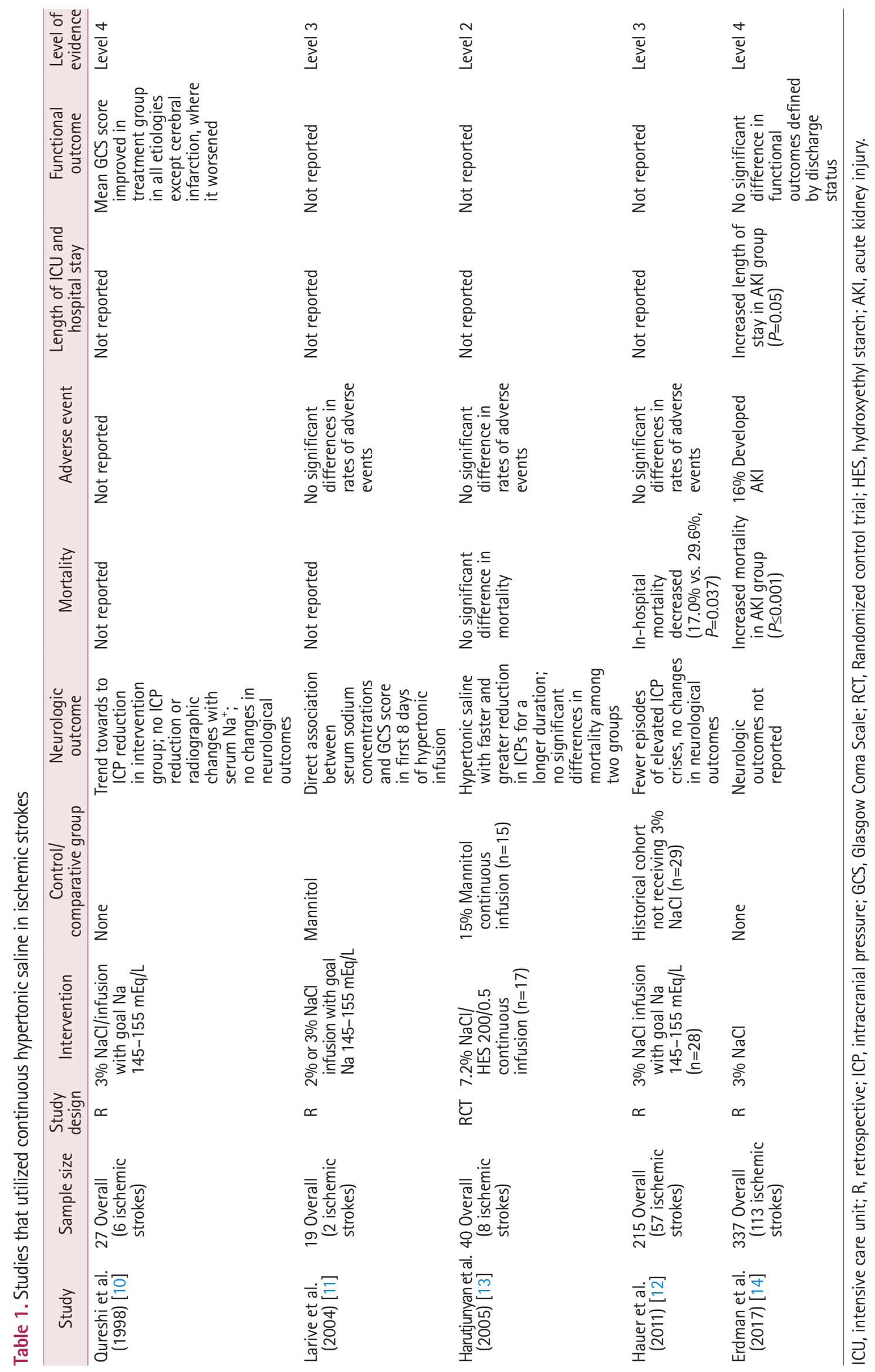


Table 2. Newcastle-0ttawa Scale for quality assessment

\begin{tabular}{|c|c|c|c|c|c|c|c|c|c|c|}
\hline \multirow{2}{*}{ Study } & \multicolumn{4}{|c|}{ Selection } & \multicolumn{2}{|c|}{ Comparability } & \multicolumn{3}{|c|}{ Outcome } & \multirow{2}{*}{ Total } \\
\hline & REC & SNEC & $\mathrm{AE}$ & DO & SC & $\mathrm{AF}$ & $\mathrm{AO}$ & FU & AFU & \\
\hline Qureshi et al. (1998) [10] & $\bigcirc$ & $\times$ & O & O & O & $x$ & O & $x$ & $\times$ & 5 (Fair) \\
\hline Hauer et al. (2011) [12] & O & O & O & O & O & $x$ & O & $x$ & $x$ & 6 (Fair) \\
\hline Larive et al. (2004) [11] & 0 & $x$ & 0 & 0 & 0 & $x$ & 0 & $x$ & $x$ & 5 (Fair) \\
\hline Erdman et al. (2017) [14] & 0 & $x$ & 0 & 0 & $x$ & $x$ & 0 & $x$ & $x$ & 4 (Fair) \\
\hline Harutjunyan et al. (2005) [13] & 0 & 0 & 0 & 0 & 0 & 0 & $\bigcirc$ & $x$ & $x$ & 7 (Good) \\
\hline
\end{tabular}

$O$, the study satisfied an item; $\times$, the study did not satisfy an item. Quality conversion: good quality (7-9), fair quality (4-6), poor quality (0-3).

REC, representativeness of the exposed cohort; SNEC, selection of the nonexposed cohort; $A E$, ascertainment of exposure; DO, demonstration that outcome of interest was not present at start of study; SC, study controls for hypertonic saline solution; AF, study controls for any additional factor; A0, assessment of outcome; FU, follow-up long enough for outcomes to occur; AFU, adequacy of follow-up cohorts.

had ischemic stroke. They investigated the effect of continuous hypertonic saline on ICP and lateral displacement in cerebral edema. Three percent hypertonic saline was infused with a target serum sodium concentration of $145-155 \mathrm{mmol} / \mathrm{L}$, with therapy continuing until patients showed improvement, complications, or lack of response. A significant correlation was observed between ICP reduction and increasing serum sodium concentration in patients with head trauma and postoperative edema but not in patients with ischemic strokes. In addition, there was no reduction in radiographic cerebral edema within 72 hours of starting the infusion. The authors proposed that low mean baseline ICP and predominance of cytotoxic edema (as opposed to primarily vasogenic edema seen in head trauma) as possible contributing factors to lack of response seen in ischemic stroke patients. Limitations of this study include the lack of a control group and small sample size [10].

In 2004, Larive et al. [11] studied 19 consecutive patients who were treated with $2 \%$ or $3 \%$ hypertonic saline - two of them with ischemic strokes. The primary objective was to assess the efficiency and time required to achieve a target serum sodium concentration of $145-155 \mathrm{mEq} / \mathrm{L}$. The secondary objective of the study was to assess the safety of hypertonic saline infusion measured by adverse effects including development of phlebitis, pneumonia, adult respiratory distress syndrome, bacteremia, sepsis, seizures, acute renal failure, arrhythmias, electrolyte disturbances, acid base disturbance, anemia, and coagulopathy. Adverse effects from the hypertonic saline cohort were compared to a mannitol cohort; however, no statistical significance between the adverse effects of the two groups was shown. Hypernatremic state was achieved after a median of 1 day; however, in some instances, it took up to 5 days to reach target hypernatremia. A significant direct association $(r=0.08, P=0.01)$ was found between the serum sodium concentrations and the Glasgow Coma Scale (GCS) score of the cohort in the first 8 days of hypertonic saline therapy. In addition, the cohort was devoid of serious adverse effects related to the hy- pertonic saline infusion. However, these results were heavily confounded by other variables and do not show clinical significance in terms of sustained neurological improvement [12].

A 2005 prospective randomized clinical study from Harutjunyan et al. [13] investigated the differential effects of efficacy and safety of $7.2 \%$ hypertonic saline infusion $(7.2 \% \mathrm{NaCl} / \mathrm{HES})$ versus $15 \%$ mannitol for 40 patients at risk of increased ICP, eight of whom had ischemic infarcts. The 40 patients were randomized to receive either $7.2 \% \mathrm{NaCl} / \mathrm{HES} 200 / 0.5$ or $15 \%$ mannitol at a defined infusion rate halted at ICPs below $15 \mathrm{mmHg}$. Primary outcomes for the study included ICP control in the respective groups. In the collective outcomes, both drugs held capabilities to lower ICP below $15 \mathrm{mmHg}$, although 7.2\% hypertonic solution showed to be more effective than mannitol. In the subgroup analysis of patients with ischemic strokes, hypertonic saline led to a faster and longer reduction in ICP compared to mannitol. However, there was no difference in mortality. Data on neurologic outcomes was not provided. The limits of the study include the absence of the rate of infusion and the non-inclusion of the targeted plasma sodium and serum osmolality levels [13].

In 2011, Hauer et al. [12] investigated the effects of continuous hypertonic saline infusion in 100 patients with a mixture of cerebrovascular diseases, including intracerebral hemorrhage, ischemic strokes, or aneurysmal subarachnoid hemorrhage. They compared patients receiving hyperosmolar therapy to a historical cohort of 115 with severe cerebrovascular disease that did not receive continuous hypertonic saline. Of these patients, 57 had ischemic infarcts. Their treatment protocol involved initiation of hypertonic saline within 72 hours of symptom onset, with adjustment of infusion rate until targets of $145-155 \mathrm{mEq} / \mathrm{L}$ and 310 $320 \mathrm{mOsm} / \mathrm{kg}$ were reached for plasma sodium and serum osmolality, respectively. Primary outcomes included frequency of ICP crises and in-hospital mortality. Overall, they observed a trend of fewer ICP crises and a significant reduction in mortality in patients who received hypertonic saline compared to controls. How- 
ever, similar to the findings of Qureshi et al. [10], these improvements were not demonstrated in those with ischemic infracts. Though identical inclusion criteria were used to identify the historical control group, the control cohort was significantly older than those receiving hypertonic saline. Age is well-established independent factor in prognosis of acute stroke and may have contributed to the survival benefit in the cohort receiving hypertonic therapy. Further, this study involved a limited cohort of patients with ischemic stroke [11].

In 2017, Erdman et al. [14] conducted a two-center retrospective cohort review to assess predictors of acute kidney injury in 337 patients receiving hypertonic saline infusion for severe neurological injuries - 113 with ischemic strokes. Acute kidney injury occurred in $16 \%$ of patients receiving hypertonic saline. In addition, by comparing those that developed an acute kidney injury with those that did not, the study found independent risk factors for developing acute kidney injury that included a history of chronic kidney disease $(P=0.007)$, serum sodium $>155 \mathrm{mmol} / \mathrm{L}$ $(P \leq 0.001)$, treatment with piperacillin/tazobactam $(P=0.002)$, male gender $(P=0.002)$, and African American race $(P=0.007)$. Interestingly, multiple risk factors commonly thought to contribute to acute kidney injury were not identified as significant in this study including mannitol, diuretics, contrast media, and other antimicrobials. In addition, type of neurologic injury (acute ischemic stroke, intracerebral hemorrhage, traumatic brain injury, or other) was not an independent risk factor. The study also noted that, because sodium concentration was recorded as a categorical variable, they could not comment on the relationship between acute kidney injury and increases in sodium. In addition, the study noted that both institutions widely use hypertonic saline infusion in patients. Thus, there was no comparison to a cohort with comparable cerebral edema that was not given hypertonic saline infusion [14].

Among the five studies, a total of 186 ischemic stroke patients were studied. In studies that involved a control group, a total of 33 patients did not receive continuous hypertonic saline. Those that received hypertonic saline were found to have fewer episodes of elevated ICP and faster and greater reduction in ICP. However, there were no significant overall differences in neurological outcomes, with some contrasting data on short-term GCS. Qureshi et al. [10] found that mean GCS worsened in ischemic stroke patients given hypertonic saline $(n=6)$, while Larive et al. [11] found a direct association between serum sodium concentrations and GCS in the first 8 days of hypertonic saline infusion $(n=2)$. The results of Larive et al. [11] may be confounded by the fact that only 2 of 19 patients in their study had ischemic strokes so GCS improvement may not be entirely applicable to this patient population. There were no significant differences in adverse events in those studies that reported this data (Table 1).

In regards to mortality, only Hauer et al. [12] found decreased mortality with use of hypertonic saline $(\mathrm{n}=28)$ by $12.6 \%$ $(P=0.037)$ when compared to control group $(n=29$ ischemic $)$. Although Erdman et al. [14] found an increased mortality $(P=0.001)$ in those that developed an acute kidney injury $(16 \%)$, patients with ischemic strokes $(n=113)$ were not at a higher risk for developing acute kidney injury. Erdman et al's study [14] was also the only study to report on length of stay and functional outcomes. They found an increase in length of stay with those that developed acute kidney injury but no overall difference in functional outcomes at discharge.

\section{DISCUSSION}

Malignant cerebral edema continues to be a prominent contributing factor to morbidity and mortality in ischemic stroke patients. Use of hypertonic therapy has been employed for decades for the reduction in cerebral edema and ICP. Although transient uses of this therapy have demonstrated reduction in ICP, no studies have demonstrated sustained reduction in cerebral edema or improvement in neurological outcomes.

Several studies have examined the effects in animal models with conflicting results. Toung et al. [4] demonstrated improvement in global cerebral edema with continuous hypertonic saline in rat models treated after MCA infarctions. Similarly, Zeng et al. [5] found that hypertonic saline was effective in reducing cerebral edema as compared to mannitol in rat models. However, Bhardwaj et al. [6] reported that hypernatremia worsened cortical infarct volume following transient focal cerebral ischemia in rats. Similarly, Papangelou et al. [7] found no reduction in brain water content on the ipsilateral hemisphere of an induced MCA stroke in rats and with no change in infarct volume.

Continuous use, however, has been sparsely studied in humans. In a review of hemispheric stroke management in 2011, Kimberly and Sheth [15] recommended eunatremic goals with no indication for administration of prophylactic hypertonic saline. Furthermore, in a 2008 review of hypertonic saline for neurologic injury, Forsyth et al. [16] highlight the lack of information on the effect of hypertonic therapy for stroke patients. These authors [16] cite several animal studies that showed hypertonic saline having a negative impact on the stroke penumbra, but data in humans is limited.

Recently published guidelines by the neurocritical care society also highlight the general lack of evidence when it comes to use of hypertonic saline for management of cerebral edema in ischemic 
strokes [17]. In addition, most studies involve comparison of mannitol versus hypertonic saline with primary outcomes being ICP control without effectively matched cohort groups. Most of these studies indicate that transient use of hypertonic saline leads to effective ICP control even in cases of mannitol failure $[13,18]$. However, use of continuous hypertonic saline has only been demonstrated in a limited number of prior studies. Our literature review has demonstrated only five studies that evaluated the role of continuous hypertonic saline-all of which failed to largely demonstrate sustained and prolonged reduction in ICP and did not appear to improve overall mortality or neurological outcomes (Table 1).

Collectively, these previous studies fail to demonstrate any significant improvement in mortality or neurological outcomes. Although several of these studies do demonstrate reduction in ICP with continuous hypertonic saline, the results are not sustained and do not translate to a clinical improvement. Additionally, lack of control groups, small sample sizes, and lack of reported data on functional outcomes and length of intensive care unit/hospital stay make these studies fairly limited in terms of generalizability. Lastly, these studies did not evaluate the role of continuous hypertonic saline in preventing or delaying decompressive hemicraniectomy surgery. Most of the studies were fair quality with relatively low levels of evidence (Tables 1 and 2). Future prospective studies with matched cohorts are needed to adequately assess the effects of continuous hypertonic saline in patients with malignant cerebral edema secondary to acute ischemic infarcts.

\section{CONCLUSION}

Continuous hypertonic saline for acute ischemic infarcts has been routinely used in neurocritical centers for the management for malignant cerebral edema. However, the data supporting its use is extremely limited. We present a systematic literature review that highlights the lack of substantial evidence supporting its use and emphasizes the study flaws that make the conclusions nongeneralizable. Although the reported studies demonstrate improvement in ICP control, there are no significant differences in neurological or functional outcomes, or overall mortality.

\section{ARTICLE INFORMATION}

\section{Ethics statement}

Not applicable.

\section{Conflict of interest}

No potential conflict of interest relevant to this article.

\section{Author contributions}

Conceptualization: AJSC, BHS, SAH. Data curation: AJSC, MM, KS, AS, KO. Formal Analysis: AJSC, MM, KS, BHS, SAH. Methodology: AJSC, KS, BHS. Visualization: AJSC, BHS, SAH. Writing-original draft: AJSC, MM, KS, BHS, AS, KO. Writing-review and editing: AJSC, BHS, SAH.

\section{REFERENCES}

1. Chen CH, Toung TJ, Sapirstein A, Bhardwaj A. Effect of duration of osmotherapy on blood-brain barrier disruption and regional cerebral edema after experimental stroke. J Cereb Blood Flow Metab 2006;26:951-8.

2. Diringer MN, Zazulia AR. Osmotic therapy: fact and fiction. Neurocrit Care 2004;1:219-33.

3. Marmarou A. A review of progress in understanding the pathophysiology and treatment of brain edema. Neurosurg Focus 2007;22:E1.

4. Toung TJ, Hurn PD, Traystman RJ, Bhardwaj A. Global brain water increases after experimental focal cerebral ischemia: effect of hypertonic saline. Crit Care Med 2002;30:644-9.

5. Zeng HK, Wang QS, Deng YY, Jiang WQ Fang M, Chen CB, et al. A comparative study on the efficacy of $10 \%$ hypertonic saline and equal volume of $20 \%$ mannitol in the treatment of experimentally induced cerebral edema in adult rats. BMC Neurosci 2010;11:153.

6. Bhardwaj A, Harukuni I, Murphy SJ, Alkayed NJ, Crain BJ, Koehler RC, et al. Hypertonic saline worsens infarct volume after transient focal ischemia in rats. Stroke 2000;31:1694-701.

7. Papangelou A, Toung TJ, Gottschalk A, Mirski MA, Koehler RC. Infarct volume after hyperacute infusion of hypertonic saline in a rat model of acute embolic stroke. Neurocrit Care 2013;18:106-14.

8. Moher D, Shamseer L, Clarke M, Ghersi D, Liberati A, Petticrew $\mathrm{M}$, et al. Preferred reporting items for systematic review and meta-analysis protocols (PRISMA-P) 2015 statement. Syst $\operatorname{Rev} 2015 ; 4: 1$

9. Ouzzani M, Hammady H, Fedorowicz Z, Elmagarmid A. Rayyan-a web and mobile app for systematic reviews. Syst Rev 2016;5:210.

10. Qureshi AI, Suarez JI, Bhardwaj A, Mirski M, Schnitzer MS, Hanley DF, et al. Use of hypertonic (3\%) saline/acetate infusion in the treatment of cerebral edema: Effect on intracranial pressure and lateral displacement of the brain. Crit Care Med 1998;26:440-6.

11. Larive LL, Rhoney DH, Parker D Jr, Coplin WM, Carhuapoma JR. Introducing hypertonic saline for cerebral edema: an aca- 
demic center experience. Neurocrit Care 2004;1:435-40.

12. Hauer EM, Stark D, Staykov D, Steigleder T, Schwab S, Bardutzky J. Early continuous hypertonic saline infusion in patients with severe cerebrovascular disease. Crit Care Med 2011;39: 1766-72.

13. Harutjunyan L, Holz C, Rieger A, Menzel M, Grond S, Soukup J. Efficiency of $7.2 \%$ hypertonic saline hydroxyethyl starch $200 / 0.5$ versus mannitol $15 \%$ in the treatment of increased intracranial pressure in neurosurgical patients: a randomized clinical trial [ISRCTN62699180]. Crit Care 2005;9:R530-40.

14. Erdman MJ, Riha H, Bode L, Chang JJ, Jones GM. Predictors of acute kidney injury in neurocritical care patients receiving continuous hypertonic saline. Neurohospitalist 2017;7:9-14.
15. Kimberly WT, Sheth KN. Approach to severe hemispheric stroke. Neurology 2011;76(7 Suppl 2):S50-6.

16. Forsyth LL, Liu-DeRyke X, Parker D Jr, Rhoney DH. Role of hypertonic saline for the management of intracranial hypertension after stroke and traumatic brain injury. Pharmacotherapy 2008;28:469-84.

17. Cook AM, Morgan Jones G, Hawryluk GW, Mailloux P, McLaughlin D, Papangelou A, et al. Guidelines for the acute treatment of cerebral edema in neurocritical care patients. Neurocrit Care 2020;32:647-66.

18. Schwarz S, Georgiadis D, Aschoff A, Schwab S. Effects of hypertonic $(10 \%)$ saline in patients with raised intracranial pressure after stroke. Stroke 2002;33:136-40. 